\title{
ON THE STRUCTURE OF CERTAIN IDEMPOTENT SEMIGROUPS
}

\begin{abstract}
BY
AHMAD SHAFAAT

Abstract. Some general theorems concerning residual finiteness of algebras are given that are applied to show that every idempotent semigroup satisfying $x y z x=x z y x$ identically is a subcartesian product of certain simple semigroups of order two and three.
\end{abstract}

Introduction. In this paper we present a technique involving a special type of infinitely long sentence which seems of fairly general applicability in the study of structure of bands (idempotent semigroups). The technique is applied to a special type of band, namely normal bands to obtain a rather complete picture of their structure. Some partial structural results on normal bands were obtained earlier by Kimura [4]. Normal bands are also considered in [8] and [2].

For a more meaningful introduction to the paper we need some definitions. A semigroup equation in a set $X$ of variables is a formula $V_{0}=V_{1}$, where $V_{0}, V_{1}$ are semigroup words in $X$, that is, finite sequences of variables chosen from $X$. A semigroup identity or law is a statement of the form $\forall x_{1}, \ldots, x_{n}(\varepsilon)$, where $\varepsilon$ is a semigroup equation. Let $R_{1}, R_{2}$ be two systems of semigroup equations in $X$ and let $\forall X\left(R_{1} \rightarrow R_{2}\right)$ denote the statement that every solution of the system $R_{1}$ in the variables of $X$ is also a solution of the system $R_{2}$. A statement of the form $\forall X\left(R_{1} \rightarrow R_{2}\right)$ is called (cf. [5]) an identical semigroup implication. The length of $\forall X\left(R_{1} \rightarrow R_{2}\right)$ by definition is the cardinal number of $R_{1}$. Identities can be regarded as implications of any given length by taking $R_{1}$ to be a big enough set of equations of the form $V=V$. Now a class $\mathscr{K}$ of semigroups is called [6] implicationally defined if $\mathscr{K}$ can be defined by a set of identical semigroup implications. If $\mathscr{K}$ can be defined by implications of finite length it is called a quasivariety. If $\mathscr{K}$ can be defined by implications of length one we call it a semivariety. Finally, if $\mathscr{K}$ is definable by implications of length zero then $\mathscr{K}$ is called a variety. All these concepts are special cases of the more general concept of a quasiprimitive class. Our arbitrary class $\mathscr{K}$ is called quasiprimitive if it is closed under the formation of isomorphs, subsemigroups and cartesian products of its semigroups. Let $Q(\mathscr{K})$ denote the class of those semigroups that are embeddable in cartesian products of

Received by the editors June 11, 1969 and, in revised form, September 22, 1969.

AMS Subject Classifications. Primary 2093, 0830.

Key Words and Phrases. Infinitely long implications, implicationally defined classes of $\Omega$-algebras, quasivarieties, semivarieties, residually finite and locally finite algebras, classes of countable (local) character, idempotent semigroups, normal idempotent semigroups. 
semigroups in $\mathscr{K}$. Then $Q(\mathscr{K})$ is the smallest quasiprimitive class containing $\mathscr{K}$; we call $Q(\mathscr{K})$ the quasiprimitive class generated by $\mathscr{K}$.

The name quasiprimitive class is borrowed from [3], where a categorical characterization of such classes is given. Implicationally defined classes are introduced in [6], where they are characterized as uniform quasiprimitive classes. (A class $\mathscr{K}$ of algebras is called uniform [6] if there exists a cardinal $\mathbb{N}$ such that an algebra $A$ is in $\mathscr{K}$ if and only if every subalgebra of $A$ generated by $\boldsymbol{N}$ elements is in $\mathscr{K}$.) Quasivarieties are well known [1] and they are precisely [6] quasiprimitive classes closed under the formation of direct limits. Varieties are also known as equational classes and are the best known of the concepts defined above. G. Birkhoff's wellknown [1] result characterizes varieties as quasiprimitive classes closed under the formation of homomorphic images. Semivarieties are introduced and characterized in [7]. Apart from the characterization of implicationally defined classes we shall not need the results mentioned in this paragraph.

The concepts of quasiprimitive classes, implicationally defined classes, quasivarieties, semivarieties and varieties become successively stronger as we go down the list. I do not know of a quasiprimitive class which is not implicationally defined although the existence of such a class is very likely. The concepts of implicationally defined classes, quasivarieties, semivarieties and varieties can however be shown to be distinct without great difficulty but we shall not concern ourselves with this here.

The last concept which we need to define for the time being is that of normal bands. A band is merely a short name for an idempotent semigroup (that is, a semigroup satisfying the identity $x^{2}=x$ ) and a band is called normal if it satisfies the identity $x y z x=x z y x$.

Our main result can now be stated as follows: Every quasiprimitive class of normal bands is a semivariety and is generated by certain easily determined semigroups of order at most 3 . This is stated later in more detail as Theorem 4 whose proof occupies $\S 2$. Other results of the paper are collected in $\S 1$ and concern algebras in general. The basic result (Theorem 1) of $\S 1$ is a straightforward generalization of the following theorem of B. H. Neumann [5]: If every countable subgroup of a group is residually finite (i.e., isomorphic to a subcartesian product of finite groups) then so is the group itself. The technique used in the proof of Theorem 4 can be outlined as follows: We first prove that all implicationally defined classes of normal bands are semivarieties. Then we apply Theorem 3 (which is applicable to bands in general) of $\S 1$ to conclude that all quasiprimitive classes of normal bands are also semivarieties. Finally using the known description of the lattice of semivarieties of normal bands we arrive at Theorem 4 .

1. Some general results and a simple application to lattices. The concepts defined above for general semigroups can be generalized to algebras in general without any difficulty. We shall use such generalizations without further mention.

Throughout this section $\mathscr{K}$ will denote an arbitrary quasiprimitive class of algebras 
with finitely many finitary operations although many of our definitions hold more generally.

Following [5] we shall say that $\mathscr{K}$ is of countable (local) character provided that an arbitrary algebra $\boldsymbol{A}$ belongs to $\mathscr{K}$ if and only if every subalgebra of $\boldsymbol{A}$ which is generated by a countable (finite) number of elements is in $\mathscr{K}$. Here 'countable' includes 'finite'. We can now state the following generalization of Neumann's theorem referred to earlier.

THEOREM 1. If $\mathscr{K}$ is generated by its finite algebras then $\mathscr{K}$ is of countable character.

We omit the proof as it follows lines of the proof of the main theorem in [5].

Our next theorem gives conditions which imply that $\mathscr{K}$ is of local character.

THEOREM 2. If $\mathscr{K}$ is generated by its finite algebras and all implicationally defined subclasses of $\mathscr{K}$ are quasivarieties then $\mathscr{K}$ is of local character.

Proof. By Theorem 1 the class $\mathscr{K}$ is of countable character. By Theorem 1 of [6] $\mathscr{K}$ is implicationally defined. By assumption $\mathscr{K}$ is a quasivariety. From the form of sentences defining a quasivariety we see immediately that every quasivariety is of local character. This proves the theorem.

Theorem 2 can be used to obtain a method of fairly wide applicability in finding implicationally defined subclasses of $\mathscr{K}$ that are not quasivarieties. This method consists in looking for locally finite algebras in $\mathscr{K}$ that are not residually finite. (An algebra is called locally finite if all of its finitely generated subalgebras are finite and is called residually finite if it is isomorphic to a subcartesian product of finite algebras.) We illustrate the method by the example of lattices.

COROLlARY 1. There are implicationally defined classes of lattices other than quasivarieties.

Proof. Let $\mathscr{L}$ be the class of all residually finite lattices. Then $\mathscr{L}$ is a quasiprimitive class generated by its finite algebras. By Theorem 2, therefore, the corollary will be proved if we show that $\mathscr{L}$ is not of local character. Consider then the lattice $L$ defined on an infinite set $L \supset\{0,1\}$ as follows:

$$
\begin{array}{ll}
x \wedge y=0 & \text { for all } x, y \in L-\{1\}, \\
x \wedge 1=x & \text { for all } x \in L, \\
x \vee y=1 & \text { for all } x, y \in L-\{0\}, \\
x \vee 0=x & \text { for all } x \in L .
\end{array}
$$

It can be easily seen that $L$ is locally finite but not residually finite $\left({ }^{1}\right)$. This shows that $\mathscr{L}$ is not of local character and the corollary is proved.

${ }^{1}$ ) An example of a lattice with this property was first pointed out to me by G. Gratzer. This particular example presented here, which is probably the simplest possible, arose in a discussion with M. Sekanina. 
For our purpose the following theorem is the most useful result of this section.

THEOREM 3. Let $\mathscr{K}$ be a quasivariety of locally finite algebras such that every implicationally defined subclass of $\mathscr{K}$ is a quasivariety. Then:

(i) Every algebra in $\mathscr{K}$ is residually finite.

(ii) Every quasiprimitive subclass of $\mathscr{K}$ is a quasivariety.

Proof. Let $\mathscr{K}$ be the class of residually finite algebras of $\mathscr{K}$. In view of local finiteness of algebras in $\mathscr{K}$, Theorem 2 shows that $\mathscr{K}=\mathscr{K}^{\prime}$. This proves (i).

Let $\mathscr{K}^{*}$ be an arbitrary quasiprimitive subclass of $\mathscr{K}$. Let $\mathscr{K}^{+}$be the quasiprimitive class generated by the finitely generated, and hence finite, algebras of $\mathscr{K}^{*}$. As in the proof of Theorem 2 we see that $\mathscr{K}^{+}$is implicationally defined. By our assumption it follows that $\mathscr{K}^{+}$is a quasivariety. We prove (ii) by showing $\mathscr{K}^{+}=\mathscr{K}^{*}$. Since $\mathscr{K}^{+}$is the quasiprimitive class generated by a subclass of the quasiprimitive class $\mathscr{K}^{*}$ we see immediately that $\mathscr{K}^{+} \subseteq \mathscr{K}^{*}$. Let $A \in \mathscr{K}^{*}$. Then finitely generated subalgebras of $\boldsymbol{A}$, being in $\mathscr{K}^{*}$, belong to $\mathscr{K}^{+}$by definition of $\mathscr{K}^{+}$. As a quasivariety $\mathscr{K}^{+}$is of local character, $\boldsymbol{A} \in \mathscr{K}^{+}$and therefore $\mathscr{K}^{*} \subseteq \mathscr{K}^{+}$. This proves the theorem.

2. Structure of normal bands. In this section we turn to the structure of normal bands. Some preliminaries are needed first.

In writing implications we shall often omit the quantifier part and write $\forall X\left(R_{1} \rightarrow R_{2}\right)$ simply as $R_{1} \rightarrow R_{2}$. This will of course notationally confuse equations with identities but there will not be any ambiguity and context will make our meaning clear.

If $R_{1}=\left\{V_{01}=V_{11}, V_{02}=V_{12}, \ldots, V_{0 m}=V_{1 m}, \ldots\right\}$ we may sometimes write $R_{1} \rightarrow R_{2}$ as $V_{01}=V_{11} \wedge \cdots \wedge V_{0 m}=V_{1 m} \wedge \cdots \rightarrow R_{2}$, specially when $R_{1}$ is finite. A similar remark holds for $R_{2}$. Clearly every implication $R_{1} \rightarrow R_{2}$ is equivalent to a set of implications of the form $R \rightarrow W_{0}=W_{1}$, one for each equation in $R_{2}$.

For a set $\Sigma$ of identical semigroup implications we shall often write [ $\Sigma$ ] for the class of all semigroups satisfying $\Sigma$.

Implications will usually be denoted by $\alpha, \beta, \gamma, \sigma$ etc., variables by $x, y, z, x_{1}, y_{1}, z_{1}$ etc., words by $U, V, W, U_{1}, V_{1}, W_{1}$ etc., and sets of variables by $X, Y, Z, X_{1}, Y_{1}, Z_{1}$ etc.

If $W=x_{1} \cdots x_{n}$ is a semigroup word we write $H(W)=x_{1}, H^{*}(W)=x_{n}$, $E(W)=\left\{x_{1}, \ldots, x_{n}\right\}$.

We define a left (right) normal band to be a band satisfying the law $x y z=x z y$ $(x y z=y x z)$. A left (right) normal band is a normal band.

The next two lemmas are aimed at showing that all implicationally defined classes of normal bands are semivarieties.

LEMMA 1. All implicationally defined classes of left (right) normal bands are semivarieties. 
Proof. We shall prove the lemma only for the left normal bands, the proof for the other case being similar. All assertions in this proof are relevant only to left normal bands. Thus if we assert that two sentences are equivalent we mean that they are equivalent for left normal bands.

To prove the lemma it is enough to show that every implication of the form

$$
\forall X\left(R \rightarrow W_{0}=W_{1}\right)
$$

is equivalent to one of length one. To this end we first recall Lemma 5.2 of [2] which states that a quasiprimitive class of left normal bands is either a variety or contains both $[x y=x]$ and $[x y=y x]$. Using this we shall assume that $[\alpha] \supseteq[x y=y]$, $[x y=y x]$ so that $\alpha$ is deducible from $x y=x$ as well as $x y=y x$. Now observe that $\alpha$ is equivalent to the three implications: $R \rightarrow W_{0}=H\left(W_{0}\right) W_{1}, R \rightarrow W_{0} W_{1}=W_{1} W_{0}$, $R \rightarrow H\left(W_{1}\right) W_{0}=W_{1}$. This shows that we have to consider only two cases according as $\alpha$ satisfies (I) $H\left(W_{0}\right)=H\left(W_{1}\right)$ or (II) $H\left(W_{0}\right) \neq H\left(W_{1}\right)$ and $E\left(W_{0}\right)=E\left(W_{1}\right)$. Since $\alpha$ is deducible from $x y=y x$ in Case I a proof of $\alpha$ from $x y=y x$ can be easily modified to obtain a proof of $\alpha$ from $x y z=x z y$ (cf. proof of Lemma 4.10 in [2]) so that $\alpha$ is equivalent to the law $x=x$. This leaves us with Case II.

Assume then that $\alpha$ satisfies $H\left(W_{0}\right) \neq H\left(W_{1}\right)$ and $E\left(W_{0}\right)=E\left(W_{1}\right)$. Let $H\left(W_{0}\right)$ $=x_{0}, H\left(W_{1}\right)=x_{1}$. Since $\alpha$ is assumed to be deducible from the law $x y=x$ it is easily seen that we can deduce from $R$ an equation of the form $x_{0} V_{0}=x_{1} V_{1}$. We complete the proof of the lemma by showing that $\alpha$ is equivalent to $x=x$ or to

$$
x_{0} V_{0}=x_{1} V_{1} \rightarrow W_{0}=W_{1} .
$$

That $\beta$ implies $\alpha$ is immediate since $R$ implies $x_{0} V_{0}=x_{1} V_{1}$. To go in the other direction let $Z$ be the set of variables $x \in X$ such that $W_{0}=V$ is deducible from $R$ for some $V$ involving $x$. Let $Z_{1}$ denote the set of $z \in Z$ such that a relation of the form $W_{1}=z W_{0}$ is deducible from $R$. Consider the substitution $s$ of the variables of $X$ defined as follows:

$$
\begin{aligned}
s(x) & =W_{1} W_{0}, & & \text { if } x \in Z_{1}, \\
& =W_{0}, & & \text { if } x \in Z-Z_{1}, \\
& =W_{0} V_{0} V_{1} W_{0}, & & \text { if } x \in X-Z .
\end{aligned}
$$

We prove that if the equation $x_{0} V_{0}=x_{1} V_{1}$ holds then the equations of $R$ hold under the substitution $s$. In other words we want to prove that if $U_{0}=U_{1}$ is in $R$ then $s\left(U_{0}\right)=s\left(U_{1}\right)$ is deducible from $x_{0} V_{0}=x_{1} V_{1}$, where for every word $U$ in $X$ we write $s(U)$ for the result of replacing $x \in E(U)$ by $s(x)$. First observe that $E\left(U_{0}\right) \subseteq Z$ if and only if $E\left(U_{1}\right) \subseteq Z$. This shows that $E\left(s\left(U_{0}\right)\right)=E\left(s\left(U_{1}\right)\right)$. If $H\left(s\left(U_{0}\right)\right)=H\left(s\left(U_{1}\right)\right)$ we clearly have $s\left(U_{0}\right)=s\left(U_{1}\right)$ without even using $x_{0} V_{0}=x_{1} V_{1}$. Let $H\left(s\left(U_{0}\right)\right)$ $\neq H\left(s\left(U_{1}\right)\right)$. From the definition of $s$ it is clear that $\left\{H\left(s\left(U_{0}\right)\right), H\left(s\left(U_{1}\right)\right)\right\} \subseteq\left\{x_{0}, x_{1}\right\}$. Without loss of generality we can suppose that $H\left(s\left(U_{0}\right)\right)=x_{0}, H\left(s\left(U_{1}\right)\right)=x_{1}$. If one of the sets $E\left(U_{0}\right), E\left(U_{1}\right)$ contains a variable from $X-Z$ then so does the other 
and $s\left(U_{0}\right)=s\left(U_{1}\right)$ is equivalent to $W_{0} V_{0} V_{1}=W_{1} V_{0} V_{1}$ which can be deduced from $x_{0} V_{0}=x_{1} V_{1}$. Hence we can assume that $E\left(U_{0}\right), E\left(U_{1}\right) \subseteq Z$. In view of the definition of $s$ we must clearly have $H\left(U_{1}\right) \in Z_{1}$. Hence we can deduce from $R$ the equation $H\left(U_{1}\right) W_{0}=W_{1}$. As $E\left(U_{1}\right) \subseteq Z$ we can easily deduce $W_{0} U_{1}=W_{0}$ from $R$ which implies the deducibility from $R$ of $H\left(U_{1}\right) U_{1} W_{0}=W_{1}$ and hence that of $H\left(U_{0}\right) U_{0} W_{0}$ $=W_{1}$, since $R$ implies $U_{0}=U_{1}$. We conclude $H\left(U_{0}\right) \in Z_{1}$ which contradicts our assumption that $H\left(s\left(U_{0}\right)\right) \neq H\left(s\left(U_{1}\right)\right)$. We have thus shown that $\alpha$ and $x_{0} V_{0}=x_{1} V_{1}$ imply $s\left(W_{0}\right)=s\left(W_{1}\right)$. If $x_{0} \notin Z$, then $s\left(W_{0}\right)=s\left(W_{1}\right)$ is equivalent to the equation $W_{0}=W_{1}$ and the equivalence of $\alpha$ and $\beta$ is proved. If $x_{0} \in Z_{1}$ we can, by the definition of $Z_{1}$, deduce $x_{0} W_{0}=W_{1}$ or $W_{0}=W_{1}$ from $R$ which implies that $\alpha$ is equivalent to the law $x=x$. The proof of the lemma is complete.

All assertions in the rest of this paper are to be considered in relation to normal bands only.

Lemma 2. All implicationally defined classes of normal bands are semivarieties.

Proof. We have to prove that the implication

$$
\forall X\left(R \rightarrow W_{0}=W_{1}\right)
$$

is equivalent to one with length one. By Lemma 4.18, Lemma (4.18)* of [2] and Lemma 1 above we can suppose that $\alpha$ is implied by every one of the laws $x y=x$, $x y=y, x y=y x$. Now it is easy to see that $\alpha$ is equivalent to the four implications

$$
\begin{aligned}
& R \rightarrow W_{0}=H\left(W_{0}\right) W_{1} H^{*}\left(W_{0}\right), \\
& R \rightarrow H\left(W_{1}\right) W_{0} H^{*}\left(W_{1}\right)=W_{1}, \\
& R \rightarrow W_{0} W_{1} W_{0}=W_{1} W_{0}, \\
& R \rightarrow W_{0} W_{1} W_{0}=W_{0} W_{1} .
\end{aligned}
$$

It is therefore enough to show that every one of $\alpha_{1}, \alpha_{2}, \alpha_{3}, \alpha_{4}$ is equivalent to an implication of length one. Since $\alpha$ is implied by $x y=y x$ so is $\alpha_{1}$. From the form of $\alpha_{1}$ it is easy to see, as before, that a proof of $\alpha_{1}$ from $x y=y x$ can be modified to yield a proof of $\alpha_{1}$ from $x y=y x$. Hence $\alpha_{1}$ and similarly $\alpha_{2}$ are equivalent separately to the law $x=x$. Next we consider $\alpha_{3}$. Since $\alpha$ is implied by $x y=x$ so is $\alpha_{3}$. It follows, as in the previous proof, that $R$ implies $x_{0} V_{0}=x_{1} V_{1}$ for some $V_{0}, V_{1}$, where $x_{0}=H\left(W_{0}\right), x_{1}=H\left(W_{1}\right)$. We can suppose that $x_{0}, x_{1}$ are distinct, for otherwise $\alpha_{3}$ can be dealt with like $\alpha_{1}$ and $\alpha_{2}$. Let $\gamma$ be the implication $x_{0} V_{0}=x_{1} V_{1} \rightarrow W_{0} W_{1} W_{0}$ $=W_{1} W_{0}$. Then the proof of equivalence of $\alpha$ and $\beta$ in Lemma 1 can be repeated without change to obtain a proof of equivalence of $\alpha_{3}$ and $\gamma$. The case of $\alpha_{4}$ is similar and the proof of the lemma is completely indicated.

Recall that $Q(\mathscr{K})$ denotes the quasiprimitive class generated by $\mathscr{K}$. If $\mathscr{K}=$ $\left\{A_{1}, A_{2}, \ldots, A_{n}\right\}$ we write $Q\left(A_{1}, \ldots, A_{n}\right)$ for $Q\left(\left\{A_{1}, \ldots, A_{n}\right\}\right)$. Recall also that for any implication $\alpha$ the class of all bands satisfying $\alpha$ is denoted by $[\alpha]$. 


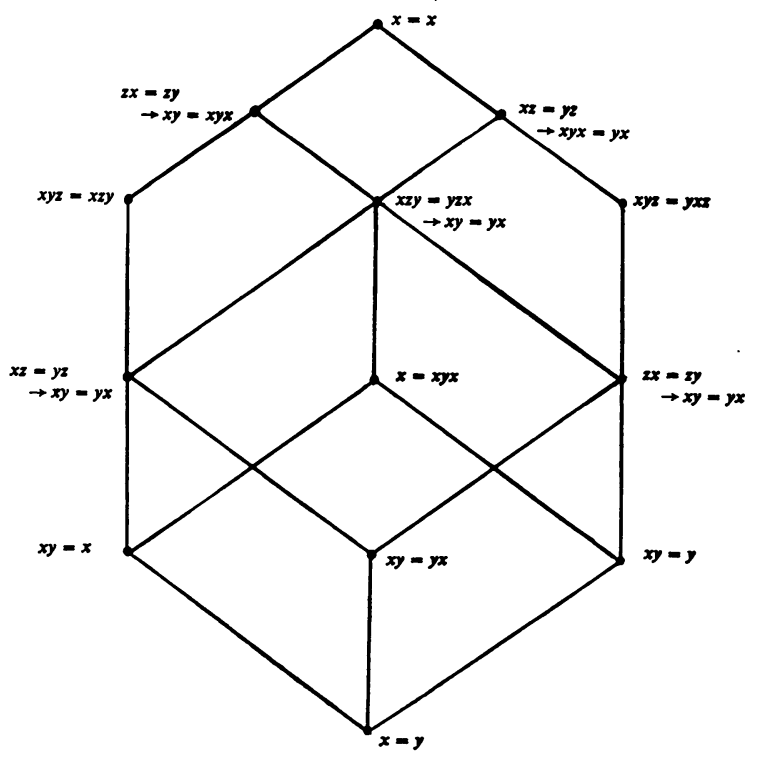

FIGURE 1

Let $S_{1}$ be the semigroup on $\{0\}$. Let $S_{2}^{-}, S_{2}^{0}, S_{2}^{+}$be the bands on $\{1,2\}$ defined respectively by: (-) $1 \cdot 2=1,2 \cdot 1=2$, (0) $1 \cdot 2=2 \cdot 1=2$, (+) $1 \cdot 2=2,2 \cdot 1=1$. Let $S_{3}^{-}, S_{3}^{+}$denote the bands obtained by adding a zero 0 to $S_{2}^{-}, S_{2}^{+}$respectively. Then we have

THEOREM 4. All quasiprimitive classes (of normal bands) are semivarieties and are precisely those given in the following list which also shows the implications defining them:

(1) $Q\left(S_{1}\right)=[x=y]$,

(2) $Q\left(S_{2}^{-}\right)=[x y=x]$,

(3) $Q\left(S_{2}^{0}\right)=[x y=y x]$,

(4) $Q\left(S_{2}^{+}\right)=[x y=y]$,

(5) $Q\left(S_{2}^{-}, S_{2}^{0}\right)=[x z=y z \rightarrow x y=y x]$,

(6) $Q\left(S_{2}^{-}, S_{2}^{+}\right)=[x y x=x]$,

(7) $Q\left(S_{2}^{0}, S_{2}^{+}\right)=[z x=z y \rightarrow x y=y x]$,

(8) $Q\left(S_{2}^{-}, S_{2}^{0}, S_{2}^{+}\right)=[x z y=y z x \rightarrow x y=y x]$,

(9) $Q\left(S_{3}^{-}\right)=[x y z=x z y]$,

(10) $Q\left(S_{3}^{+}\right)=[x y z=y x z]$,

(11) $Q\left(S_{3}^{-}, S_{2}^{+}\right)=[z x=z y \rightarrow x y x=x y]$,

(12) $Q\left(S_{3}^{+}, S_{2}^{-}\right)=[x z=y z \rightarrow x y x=y x]$,

(13) $Q\left(S_{3}^{-}, S_{3}^{+}\right)=[x=x]$.

Proof. First note that normal bands are locally finite. This follows from the easily verifiable fact that under idempotency and normality every semigroup 
word in $n$ variables is congruent to one of length at most $n+2$. In view of local finiteness of normal bands Theorem 3 and Lemma 2 immediately show that all quasiprimitive classes of normal bands are semivarieties. Now the lattice of semivarieties of normal bands is known [2] and is described by Figure 1.

Now (1) is trivial and (2)-(7) $\left({ }^{2}\right)$ can be read immediately by Figure 1 . To see (8) first note that $S_{3}^{-}$is left normal band which does not satisfy $x z=y z \rightarrow x y=y x$ (take $x=1, y=2, z=0$ ). Moreover $S_{2}^{-}, S_{2}^{0}$ are embeddable in $S_{3}^{-}$. Hence from (5) $[x z=y z \rightarrow x y=y x] \subset Q\left(S_{3}^{-}\right) \subseteq[x y z=x z y]$. Figure 1 then immediately says that $[x y z=x z y]=Q\left(S_{3}^{-}\right)$. Proofs of the equalities (9)-(13) are similar and are omitted.

We state parts of the above theorem in slightly different forms in the following corollaries that are immediate from the fact that $S_{2}^{-}, S_{2}^{0}\left(S_{2}^{+}, S_{2}^{0}\right)$ are the only nontrivial subsemigroups of $S_{3}^{-}\left(S_{3}^{+}\right)$.

COROLlARY 2. Every normal band is a subcartesian product of some of the bands $S_{2}^{-}, S_{2}^{0}, S_{2}^{+}, S_{3}^{-}, S_{3}^{+}$.

COROLlaRY 3. Every left (right) normal band is a subcartesian product of some of the bands $S_{2}^{-}, S_{2}^{0}, S_{3}^{-}\left(S_{2}^{+}, S_{2}^{0}, S_{3}^{+}\right)$.

\section{REFERENCES}

1. P. M. Cohn, Universal algebra, Harper and Row, New York, 1965. MR 31 \#224.

2. J. A. Gerhard and Ahmad Shafaat, Semivarieties of idempotent semigroups (prepublication copy).

3. J. R. Isbell, Subobjects, adequacy, completeness and categories of algebras, Rozprawy Mat. 36 (1964). MR 29 \#1238.

4. Naoki Kimura, Note on idempotent semigroups. II, Proc. Japan. Acad. 34 (1958), 110-112.

5. B. H. Neumann, Group properties of countable character (prepublication copy).

6. Ahmad Shafaat, Implicationally defined classes of algebras, J. London Math. Soc. 44 (1969), 137-140. MR 38 \#5691.

7. —_ Characterizations of some universal classes of algebras, J. London Math. Soc. (to appear).

8. T. Tamura, Attainability of systems of identities on semigroups, J. Algebra 3 (1966), 261-276. MR 32 \#7668.

CARLeton University, Ottawa, Ontario, Canada

(2) The equalities (2)-(4) are well known. 